\title{
What does reflection from cloud sides tell us about vertical distribution of cloud droplet sizes?
}

\author{
A. Marshak ${ }^{1}$, J. V. Martins ${ }^{1,2}$, V. Zubko ${ }^{3,4}$, and Y. J. Kaufman ${ }^{1}$ \\ ${ }^{1}$ NASA - Goddard Space Flight Center, Climate and Radiation Branch, Greenbelt, MD, USA \\ ${ }^{2}$ Joint Center for Earth Systems Technology, University of Maryland Baltimore County, Baltimore, MD, USA \\ ${ }^{3}$ RS Information Systems, Inc., Greenbelt, MD, USA \\ ${ }^{4}$ NASA - Goddard Space Flight Center, Goddard Earth Sciences Data and Information Services Center, Greenbelt, MD, USA
}

Received: 2 May 2006 - Published in Atmos. Chem. Phys. Discuss.: 31 July 2006

Revised: 8 November 2006 - Accepted: 8 November 2006 - Published: 20 November 2006

\begin{abstract}
Cloud development, the onset of precipitation and the effect of aerosol on clouds depend on the structure of the cloud profiles of droplet size and phase. Aircraft measurements of cloud profiles are limited in their temporal and spatial extent. Satellites were used to observe cloud tops not cloud profiles with vertical profiles of precipitation-sized droplets anticipated from CloudSat. The recently proposed CLAIM-3D satellite mission (cloud aerosol interaction mission in 3-D) suggests to measure profiles of cloud microphysical properties by retrieving them from the solar and infrared radiation reflected or emitted from cloud sides.

Inversion of measurements from the cloud sides requires rigorous understanding of the 3-dimentional (3-D) properties of clouds. Here we discuss the reflected sunlight from the cloud sides and top at two wavelengths: one nonabsorbing to solar radiation $(0.67 \mu \mathrm{m})$ and one with liquid water efficient absorption of solar radiation $(2.1 \mu \mathrm{m})$. In contrast to the plane-parallel approximation, a conventional approach to all current operational retrievals, 3-D radiative transfer is used for interpreting the observed reflectances. General properties of the radiation reflected from the sides of an isolated cloud are discussed. As a proof of concept, the paper shows a few examples of radiation reflected from cloud fields generated by a simple stochastic cloud model with the prescribed vertically resolved microphysics. To retrieve the information about droplet sizes, we propose to use the probability density function of the droplet size distribution and its first two moments instead of the assumption about fixed values of the droplet effective radius. The retrieval algorithm is based on the Bayesian theorem that combines prior information about cloud structure and microphysics with radiative transfer calculations.
\end{abstract}

Correspondence to: A. Marshak

(alexander.marshak@nasa.gov)

\section{Introduction}

Investigation of cloud development and the onset of precipitation are essential to understand the role of clouds in the hydrological cycle and the effect of pollutants on clouds and precipitation (Ramanathan et al., 2001). It also advances our understanding of the feedback of clouds on climate change and the aerosol indirect forcing of climate through cloud modification. Therefore, we have to resolve the vertical distribution of cloud droplet sizes and determine the temperature of glaciation for clean and polluted clouds (Andreae et al., 2004). Knowledge of the droplet vertical profile is also essential for understanding precipitation (Rosenfeld and Lensky, 1998; Rosenfeld, 2000; Rosenfeld and Ulbrich, 2000). In an accompanied paper, Martins et al. $(2006)^{1}$ suggest a satellite mission to derive profiles of the cloud microphysics using observations of the cloud sides. Here we show a methodology, based on 3-dimensional (3-D) cloud properties to retrieve the cloud profiles from the new satellite measurements.

So far, all existing satellites either measure cloud microphysics only at cloud top (e.g., Moderate Resolution Imaging Spectrometer (MODIS), see Platnick et al., 2003) or give a vertical profile of precipitation sized droplets (e.g., Tropical Rainfall Measuring Mission (TRMM) Precipitation Radar, see Hirose and Nakamura, 2004). Note that the combination of millimeter-wave radar reflectivity measured by recently launched CloudSat (Stephens et al., 2002) with MODIS (on Aqua) measurements of solar radiance is able to provide cloud droplet size vertical profiles but under some strong assumptions of given number concentration and droplet size distribution.

\footnotetext{
${ }^{1}$ Martins, J. V., Marshak, A., Remer, L., Rosenfeld, D., Kaufman, Y. J., Fernandez-Borda, R., Koren, I., Zubko, V., and Artaxo, P.: Remote sensing the vertical profile of cloud droplet effective radius, thermodynamic phase, and temperature, Atmos. Chem. Phys. Discuss., submitted, 2006.
} 
Except for Polarization and Directionality of the Earth's Reflectance (POLDER) that retrieves cloud droplet effective radius at the very top cloud layer (with an optical thickness of 1) from polarization measurements of the reflected light (e.g., Breon and Golub, 1998, Breon and Doutriaux-Boucher, 2005), all operational retrievals of cloud droplet size are based on spectral observations (e.g., Nakajima and King, 1990). For MODIS, cloud optical thickness, $\tau$, and droplet effective radius, $r_{e}$, are simultaneously derived from various two band combinations: typically one water-absorbing band $\{1.6,2.1$, or $3.7 \mu \mathrm{m}\}$ and one nonabsorbing band $\{0.65,0.86$, or $1.2 \mu \mathrm{m}$ \} (Platnick et al., 2003). Since water absorbs differently in the three MODIS absorbing bands, the less absorbing 1.6- $\mu \mathrm{m}$ band and the more absorbing 3.7- $\mu \mathrm{m}$ band complement to the $2.1-\mu \mathrm{m}$ band for assessing the vertical variation of $r_{e}$ in the upper portion of the cloud (Platnick, 2000; Chang and $\mathrm{Li}, 2002$ ). However, these variations are not sufficient to resolve the vertical distribution of cloud droplet sizes from cloud base to cloud top. What is if one would measure the vertical profiles of the cloud microphysical properties by retrieving them from the solar (and infrared) radiation reflected (or emitted) directly from cloud sides?

Note that all existing operational retrieval algorithms are based on the plane-parallel approximation that does not take into account the cloud horizontal inhomogeneity. In terms of cloud aspect ratio, $A=L / h$ (where $L$ and $h$ are horizontal and vertical dimensions of a cloud, respectively), the main planeparallel assumption used for any remote sensing retrieval is that $A$ is infinitely large and that the satellite always sees the cloud top. Hence, a pair of reflectances at the nonabsorbing and absorbing bands indicates how optically thick (thus estimates $\tau$ ) and how absorbing (thus estimates $r_{e}$ ) clouds are (Nakajima and King, 1990).

It is well understood that finite isolated clouds of various shapes and sizes can have absolutely different radiative properties than their plane-parallel counterparts. Davies (1978) represented an isolated cloud as a cuboid of given dimensions. In this case, the incident solar beam hits not only the top of the cloud but also one or two of its sides. As an alternative to the plane-parallel model to simulate cumulus clouds, recently Davis (2002) used a spherical turbid medium. For his spherical cloud, he was able to derive analytically the transmitted and reflected fluxes in terms of the cloud optical diameter. He showed that these results could be used to estimate the cloud optical diameter from radiances reflected from dark and bright sides of clouds.

In general, if one releases the assumption that the aspect ratio $A$ is infinitely large then, in addition to cloud tops, a satellite-based observer will likely see cloud sides. Because of a variety of possible aspect ratios and cloud geometrical shapes, the situation seems to be out of control and measured data cannot be correctly interpreted in the sense of cloud properties. Similar to the plane-parallel approximation, in order to bring the retrieval back under control we have to make simplifying assumptions. The main assumption for cloud side remote sensing is that regardless of the aspect ratio, cloud geometrical shape and its microphysical structure, a pair of reflectances at nonabsorbing and absorbing bands determines a distribution of droplet sizes. Note that this is an assumption rather than a statement since it can't be checked with the model calculation and inversion for all cloud types. Also note that here we are talking about the distribution of droplet sizes (with mean and standard deviation) rather than a single value. Finally, together with the brightness temperature this assumption allows us to estimate a vertical profile of droplet (particle) sizes (Martins et al., 2006 ${ }^{1}$ ).

Of course, the above assumption will not work for all cloud types like the plane-parallel approximation does not work for all clouds either. Here we will consider only optically thick clouds $(\tau \geq 40)$ with relatively small aspect ratio $(L / h \leq 2-5)$. We will further make some additional limitations regarding the satellite viewing angles. In order to see a sufficient amount of cloud sides, the viewing zenith angles, $\theta$, will be limited to more oblique angles of $\theta \geq 45^{0}$. For simplicity here we will be considering only "backward" directions, i.e., $\varphi \approx \varphi_{0}$ where $\varphi_{0}$ and $\varphi$ are solar and viewing azimuthal angles, respectively. Under these rather strong limitations, the paper proves the concept of a possible retrieval of the distributions of droplet vertical profiles using three bands: nonabsorbing, water absorbing and brightness temperature. The latter is associated with the measured height and is discussed in the companion paper (Martins et al., 2006 ${ }^{1}$ ).

Another assumption for cloud side remote sensing used here is that the vertical profiles of droplet size distributions retrieved at cloud sides are representative for the whole cloud. Indeed, for a given temperature (and non precipitating clouds) droplet effective radius, $r_{e}$, is, perhaps, the most stable and robust microphysical parameter (see Rosenfeld and Lensky, 1998; Freud et al., 2005). To study the evolution of $r_{e}$ of convective clouds particles with temperature, Rosenfeld and Lensky (1998) developed the methodology that identifies different microphysical processes at different heights. They found that the temperature versus the effective radius relations provide significant information about precipitation forming processes in convective clouds.

The plan of the paper is as follows. Section 2 briefly discusses the main radiative transfer features of the reflectance from cloud sides based on a single homogeneous cloud. To generalize these results to a more realistic horizontally inhomogeneous cloud field, Sect. 3 describes simple stochastic and microphysical models used to simulate a variety of cloud fields. With the help of two wavelengths at 0.67 and $2.1 \mu \mathrm{m}$, Sect. 4 demonstrates the retrievals of the distribution of droplet sizes from the measurements of radiation reflected from the cloud fields simulated in Sect. 3. At the end of Sect. 4, this approach is generalized in the terms of Bayesian retrievals (McFarlane et al., 2002; Evans et al., 2002). Finally Sect. 5 provides general discussion and summarizes the results. 


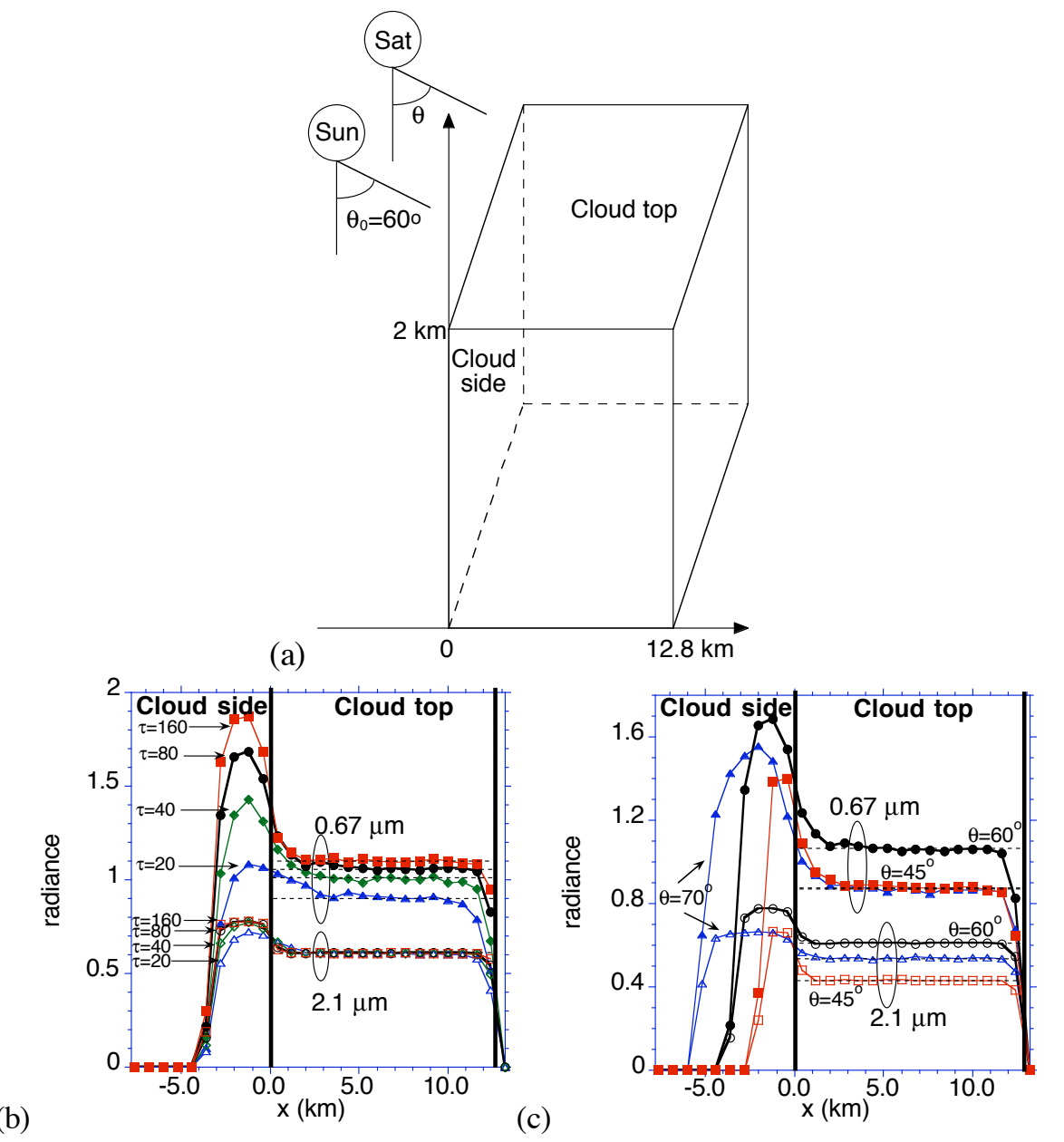

Fig. 1. Reflectance from a single cloud at two wavelengths: $0.67 \mu \mathrm{m}$ (solid symbols) and $2.1 \mu \mathrm{m}$ (empty symbols). Cloud height $h=2 \mathrm{~km}$, cloud width $L=12.8 \mathrm{~km}$, droplet effective radius, $r_{e}=10 \mu \mathrm{m}, \theta_{0}=60^{\circ}$ surface is absorbing. (a) A schematic illustration of illumination (Sun) and viewing (Sat) angles. Negative $x$ correspond to reflectances from "cloud side" while positive $x$ correspond to reflectances from "cloud top"; (b) $\theta=60^{\circ}$; cloud optical thickness $\tau=160,80,40$ and 20 ; (c) $\tau=80, \theta=70^{\circ}, 60^{\circ}$ and $45^{\circ}$.

\section{Radiative transfer calculations}

\subsection{3-D radiative transfer tools}

There are two 3-D Radiative Transfer (RT) tools that dominate atmospheric radiation applications and are currently the only available options for solving complex RT problems: the Spherical Harmonic Discrete Ordinate Method (SHDOM) of Evans (1998) and the Monte Carlo (MC) method (Marchuk et al., 1980). When many radiative quantities are required, e.g., the radiance field across cloud top, SHDOM is much faster than MC, but its errors (and limitations) are harder to interpreter, especially for optically thick and highly variable media around cloud edges. Since the rule-of-thumb in using SHDOM requires the optical path across a grid cell to be of order of one, its solution may be not accurate for horizontally and vertically thick clouds. Moreover, SHDOM (tri)linearly interpolates the extinction between grid points; thus it may have some problems when reflectance from cloud sides of optically thick clouds is calculated. Anyway, in this study we used both MC and SHDOM; for several key calculations both methods were applied simultaneously to the same set of cloud parameters to intercompare and validate the results. To the best of our knowledge, the results shown in the paper are numerically accurate. (For the detailed description of both the SHDOM and MC methods, see Evans and Marshak, 2005).

\subsection{Main radiative transfer features of the reflectance from cloud sides}

Using a 3-D Monte Carlo radiative transfer code (Evans and Marshak, 2005), we calculated reflectance at 0.67 and $2.1 \mu \mathrm{m}$ wavelengths from a single homogeneous cloud. The cloud top was simulated by an infinitely long rectangular shape with width $L=12.8 \mathrm{~km}$ and height $h=2 \mathrm{~km}$ (Fig. 1a). 


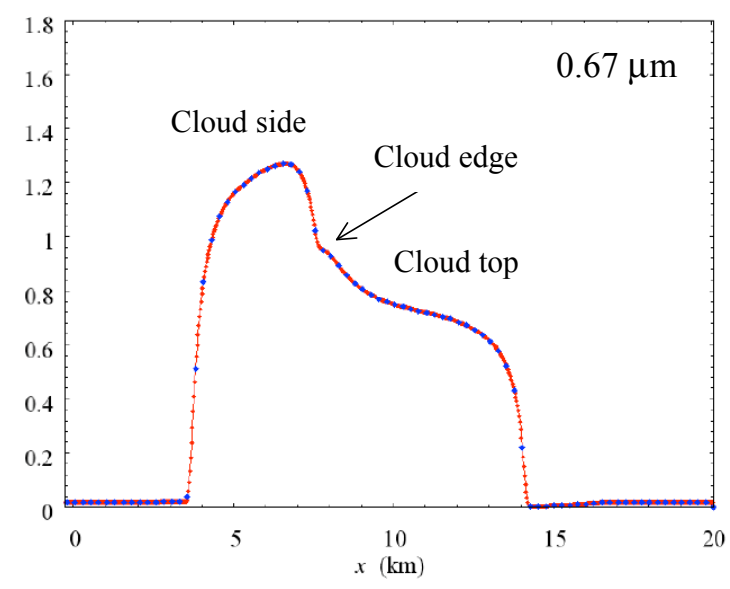

(a)

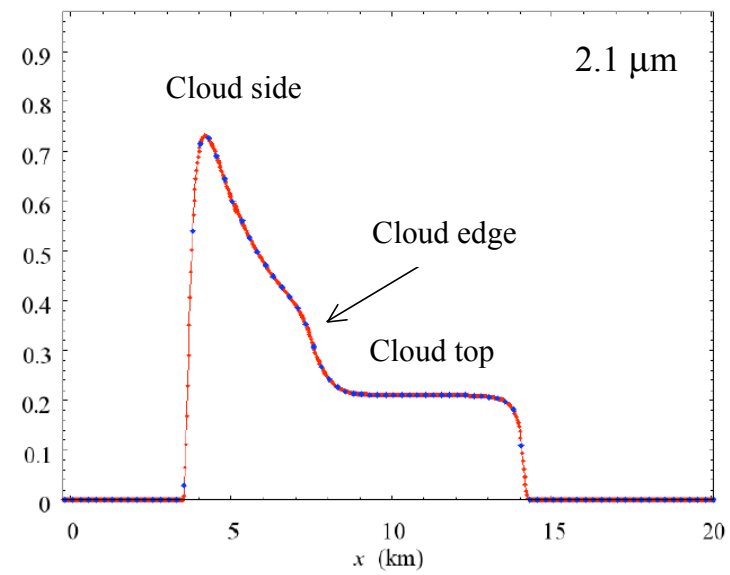

(b)

Fig. 2. Reflectance from a single cloud with a variable droplet effective radius. Cloud height $h=4 \mathrm{~km}$, cloud width $L=6.5 \mathrm{~km}$, flat cloud top, $\tau=80, \theta_{0}=60^{\circ}, \theta=45^{\circ}$. Droplet effective radius $r_{e}$ increases linearly with height from 5 to $25 \mu \mathrm{m}$. Cloud edge is indicated by arrow at $x=7.5 \mathrm{~km}$. Reflectance from cloud top is at the right side from the cloud edge while reflectance from cloud side is at the left. Dots indicate "measurements" at $s=0.25 \mathrm{~km}$ resolution. (a) $0.67 \mu \mathrm{m}$; (b) $2.1 \mu \mathrm{m}$.

Cloud vertical optical thickness, $\tau$, varied from 20 to 160 and droplet effective radius was assumed a constant $r_{e}=10 \mu \mathrm{m}$. (Here and through the whole paper, surface is assumed absorbing.) The cloud was illuminated at solar zenith angle (SZA) $\theta_{0}=60^{\circ}$ along cloud inhomogeneity and observed at viewing zenith angles (VZA) $\theta=45^{\circ}-70^{\circ}$ from the illuminated side of the cloud. The reflectances are plotted in panels (b) and (c) on Fig. 1. The horizontal axis shows the distance to the cloud edge (negative $x$-values) and the distance from the cloud edge (positive $x$-values). The cloud edge is located at $x=0$. For example, a cloud side, viewed at $\theta=70^{\circ}$, can be seen $h \times \tan (\theta)=5.5 \mathrm{~km}$ away from the cloud (negative $5.5 \mathrm{~km}$ ). Thus negative $x$-values correspond to radiation reflected from a cloud side while positive $x$-values to radiation reflected from a cloud top. Here are the main features that can be observed from these two panels.
- Reflectance from a cloud side at $2.1 \mu \mathrm{m}$ is saturated starting from $\tau=40$ while reflectance at $0.67 \mu \mathrm{m}$ does not reach the level of saturation at all or will be saturated only at very large values of cloud optical thickness $\tau$. The maximum $2.1 \mu \mathrm{m}$ reflectance from cloud sides, $I_{\text {side }}\left(\theta_{0}, \theta\right)$, depends on both $\theta_{0}$, and $\theta$. It can be estimated as

$I_{\text {side }}\left(\theta_{0}, \theta\right) \leq I_{p p}\left(90^{\circ}-\theta_{0}, 90^{\circ}-\theta, \varphi-\varphi_{0}=180^{\circ}, \tau=\infty\right)$

$\cos \left(90^{\circ}-\theta_{0}\right) / \cos \left(\theta_{0}\right)$,

where $I_{p p}\left(\theta_{0}, \theta, \varphi-\varphi_{0}, \tau\right)$ is the cloud top reflectance calculated using the plane-parallel approximation (Stamnes et al., 1988). For example, for $\theta_{0}=\theta=60^{\circ}$, the $2.1 \mu \mathrm{m}$ reflectance

$I_{\text {side }}\left(60^{\circ}, 60^{\circ}\right) \leq I_{p p}\left(30^{\circ}, 30^{\circ}, \varphi-\varphi_{0}=180^{\circ}, \tau=160\right)$

$\cos \left(30^{\circ}\right) / \cos \left(60^{\circ}\right)=0.782$,

as seen in panel $b$.

- The more oblique viewing zenith angle $\theta$ (or the larger cloud side, $h$ ) the wider the area of maximal reflectance at $2.1 \mu \mathrm{m}$ (panel c).

- For optically and geometrically thick clouds, the reflectance from cloud side near cloud top is smaller than the one reflected from the middle of the cloud side. This effect is much more pronounced for $0.67 \mu \mathrm{m}$ than for $2.1 \mu \mathrm{m}$.

- For thick clouds, starting from a few optical depths away from cloud edges, reflectance from cloud top at $2.1 \mu \mathrm{m}$ is well approximated by the plane-parallel approximation. Depending on the extinction coefficient, it is not always the case for reflectance at $0.67 \mu \mathrm{m}$. At both wavelengths reflectance from cloud top increases towards the illuminated side and decreases towards the shadowed side.

- Finally, the number of measurements from cloud side is equal to $h \times \tan (\theta) / s$ where $s$ is the horizontal resolution of a radiometer. For example, if $h=2 \mathrm{~km}, \theta=70^{\circ}$, and $s=0.1 \mathrm{~km}$, there will be 55 cloud side measurements.

All of the above radiative transfer features will be observed by analyzing the reflectance from more complex cloud fields.

\subsection{Reflectance from cloud sides for a cloud with variable droplet sizes}

Figure 2 shows an example of reflectances from cloud side and cloud top for the same two wavelengths $(0.67 \mu \mathrm{m}$ and $2.1 \mu \mathrm{m})$ but with droplet effective radius $r_{e}$ increasing linearly with height from $5 \mu \mathrm{m}$ (at the cloud base) to $25 \mu \mathrm{m}$ (at the cloud top). Cloud geometrical thickness $h=4 \mathrm{~km}$ and cloud optical thickness is $\tau=80$ (thus extinction coefficient is 
$20 \mathrm{~km}^{-1}$ ). With horizontal resolution $s=0.25 \mathrm{~km}$ and VZA $\theta=45$, there are $h \times \tan (\theta) / s=16$ cloud side "measurements." As for a simple example in Fig. 1, $I_{0.67}$ reaches its maximum near cloud top (actually about $1 \mathrm{~km}$ from the cloud top) where yet most of the photons are reflected back from the cloud side without either transmitting through cloud and escaping from cloud base or reflecting from cloud top. Unlike in the previous example, the horizontal size $L$ of a cloud is only $6.5 \mathrm{~km}$ and with the extinction coefficient $20 \mathrm{~km}^{-1}$ this is not sufficient to reach a stable plane-parallel regime at cloud top. As a result, $I_{0.67}$ keeps decreasing from the illuminated cloud edge to the shadowed one. In contrast, $I_{2.10}$ has a flat plateau of $5 \mathrm{~km}$ across where the 3-D reflectance perfectly matches the plane-parallel one. Because of increasing droplet sizes with height, the maximum is reached much lower than in case of conservative scattering. It is around $1 \mathrm{~km}$ from cloud base where $r_{e}=9-11 \mu \mathrm{m}$. With farther increase of $r_{e}$, reflectance $I_{2.10}$ drops fast and reaches a flat plane-parallel level already at the cloud top $\left(r_{e}=25 \mu \mathrm{m}\right)$ about $1 \mathrm{~km}$ from the cloud edge.

The study of reflectance from an isolated finite-size cloud is not new and has begun yet in early 70s (see, e.g., McKee and Cox, 1974; Davies, 1978 and 1984). As it is seen from Figs. 1 and 2, cloud side reflectances at the two (water-absorbing and nonabsorbing) wavelengths, have welldetermined features. Not unlike their cloud top counterparts in the plane-parallel approximation (Nakajima and King, 1990), the combination of these two reflectances can be mapped into retrievals of cloud optical $(\tau)$ and microphysical $\left(r_{e}\right)$ structure. The key question here is whether these features survive if applied to realistic cloud fields rather than a single isolated horizontally homogeneous cloud. Next we briefly discuss cloud models used in this study.

\section{Cloud models}

Realistic 3-D cloud fields, as an input in radiative transfer calculations, can be obtained from either dynamical or stochastic cloud models. For the purpose of this paper (to learn what reflection from cloud sides tells us about vertical distribution of cloud particles), a choice of model is not very crucial. The main requirements for a model were set as to have a field of several joined and disjoined clouds with the prescribed (observed) mean, standard deviation and correlation function of variable cloud optical thickness $\tau(x, y)$ with a desired cloud fraction $A_{c}$ and cloud top height $h(x, y)$. Having some experience in stochastic cloud modeling (e.g., Marshak et al., 1994; Prigarin and Marshak, 2005), we selected a broken cloud version (Marshak et al., 1998) of a simple fractionally integrated cascade model (Schertzer and Lovejoy, 1987) that generates cloud fields with a given power spectral exponent, mean and standard deviation of cloud optical thickness. To correlate $\tau(x, y)$ with $h(x, y)$, we generated independently a $\tau(x, y)$-field and the mean photon free

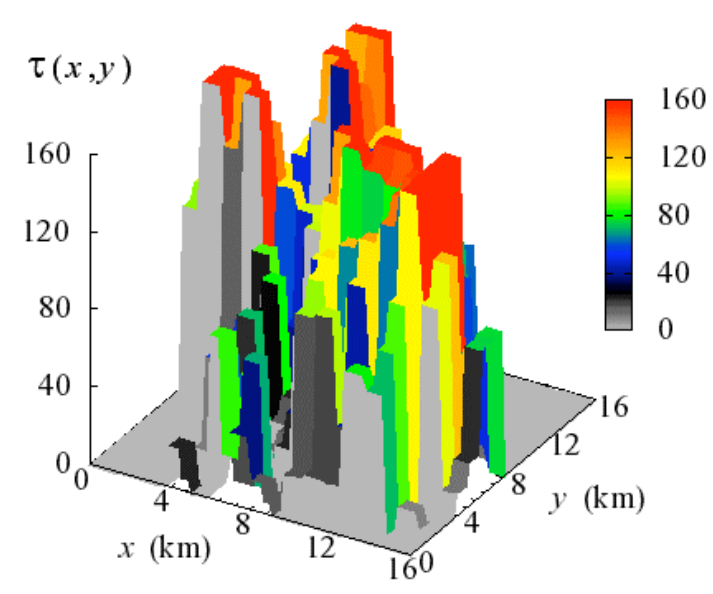

(a)

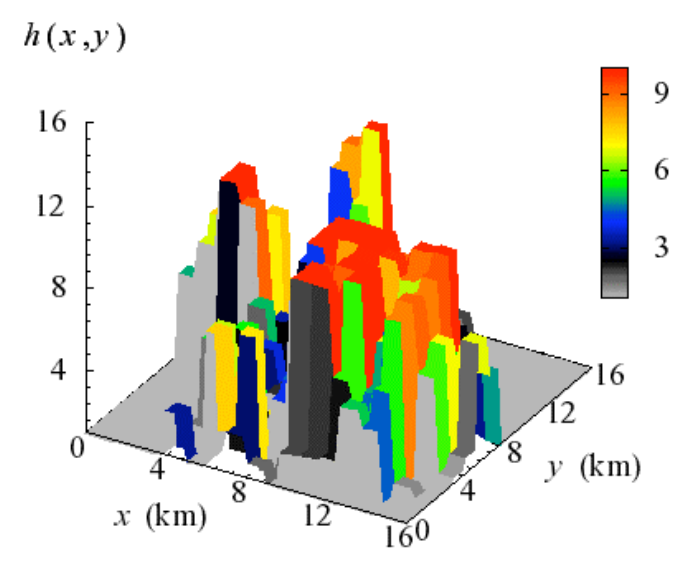

(b)

Fig. 3. A realization of cloud stochastic model that has a given power-spectral exponent, mean, and standard deviation. (a) optical depth filed; (b) cloud top height field.

path field $l(x, y)$. The cloud geometrical thickness field (assuming cloud base to be a constant) is thus a product between the optical depth and the mean free path fields,

$h(x, y)=\tau(x, y) * l(x, y)$.

Figure 3 illustrates one realization of a cloud with an array of optical and geometrical thicknesses. Though it might not look very realistic, it preserves the observed correlation function in both optical and geometrical thicknesses.

After cloud structure, cloud microphysics is perhaps the most important cloud model feature needed for radiative transfer calculations. For simplicity and for more 
LWC

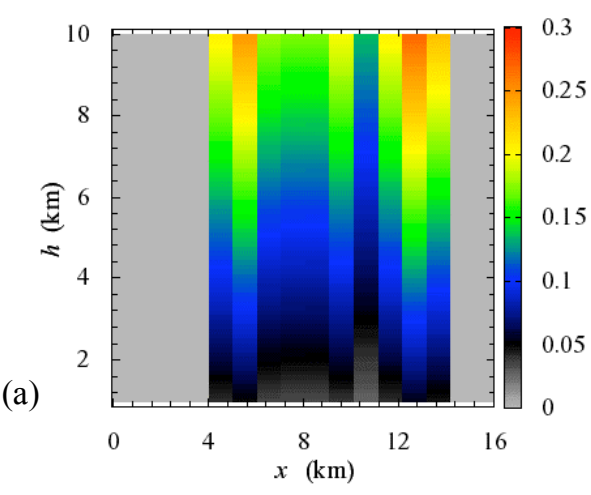

(c)

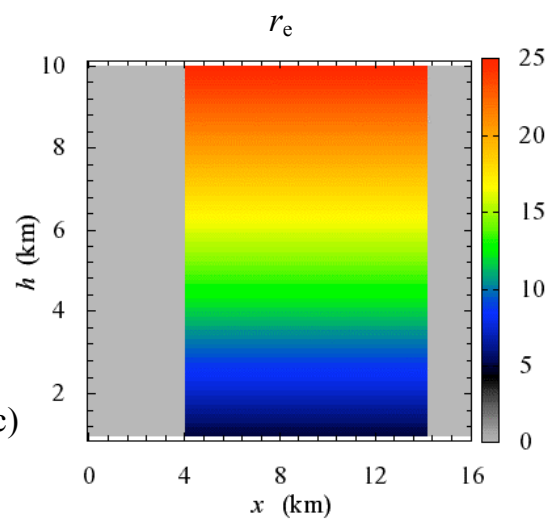

$N$
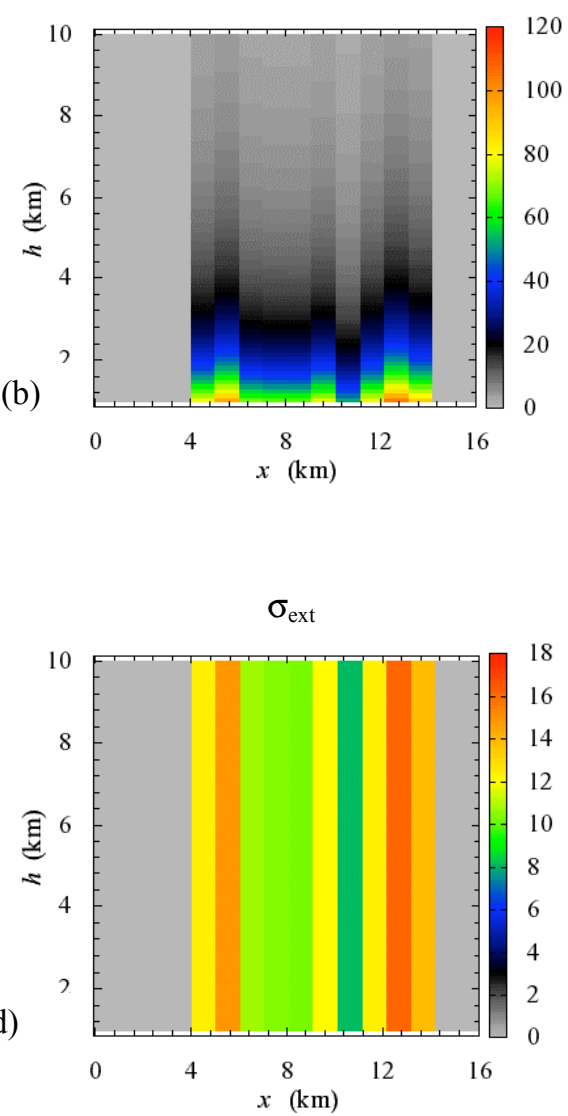

Fig. 4. An example of cloud microphysics. (a) liquid water content, LWC; (b) number of drops, $N$; (c) droplet effective radius, $r_{e}$; (d) extinction coefficient, $\sigma_{\text {ext }}$.

straightforward interpretation of the simulated radiative transfer results, we made two assumptions:

- cloud droplets grow linearly with $z$, i.e.,

$r_{e}(z ; x, y)=a\left(z-z_{0}\right)+b, z_{0} \leq z \leq h(x, y)$,

$a$ and $b$ are constants

- the extinction coefficient $\sigma_{\text {ext }}$ does not depend on $z$, i.e.,

$\sigma_{\text {ext }}(z ; x, y) \equiv \sigma_{\text {ext }}(x, y)$.

Note that under some general assumptions (e.g., Platnick, 2000), cloud liquid water content (LWC) is proportional to a product of the density of liquid water, $\rho$, cube of the droplet effective radius, $r_{e}$, and the total number of droplets in unit volume, $N$,

$L W C(z ; x, y) \approx \frac{4}{3} \pi \rho r_{e}^{3}(z ; x, y) N(z ; x, y)$.
Cloud LWC is also related to $\tau, r_{e}$, and $\rho$ as (Stephens, 1994, p. 219)

$\tau(x, y)=\frac{3}{2 \rho} \int_{0}^{h(x, y)} \frac{L W C(z ; x, y)}{r_{e}(z ; x, y)} d z$.

Therefore, with the assumptions (3a)-(3b), $N$ changes with vertical coordinate $z$ as $r_{e}^{-2}$, namely,

$N(z ; x, y)=\frac{\tau(x, y)}{2 \pi h(x, y)} \frac{1}{r_{e}^{2}(z ; x, y)}=\frac{1}{2 \pi} \frac{\sigma_{\mathrm{ext}}(x, y)}{r_{e}^{2}(z ; x, y)}$. (5a)

At the cloud base for $z=z_{0}$, we get

$N\left(z_{0} ; x, y\right)=\frac{1}{2 \pi} \frac{\sigma_{\mathrm{ext}}(x, y)}{b^{2}}$.

For example, for $r_{e}\left(z_{0}\right)=b=5 \mu \mathrm{m}$ and $\sigma_{\mathrm{ext}}=20 \mathrm{~km}^{-1}$ one gets $N\left(z_{0}\right)=127 \mathrm{~cm}^{-3}$. If at the cloud top $r_{e}=25 \mu \mathrm{m}$ then Eq. (5a) yields $N\left(z_{0}\right)=5 \mathrm{~cm}^{-3}$. Figure 4 shows an example of vertical profiles for cloud liquid water, LWC (in $\mathrm{g} / \mathrm{m}^{3}$ ), total number of drops, $N$ (in $\mathrm{cm}^{-3}$ ), effective radius, $r_{e}($ in $\mu \mathrm{m}$ ), and extinction coefficient, $\sigma_{\text {ext }}\left(\right.$ in $\left.\mathrm{km}^{-1}\right)$. We see that, for each 

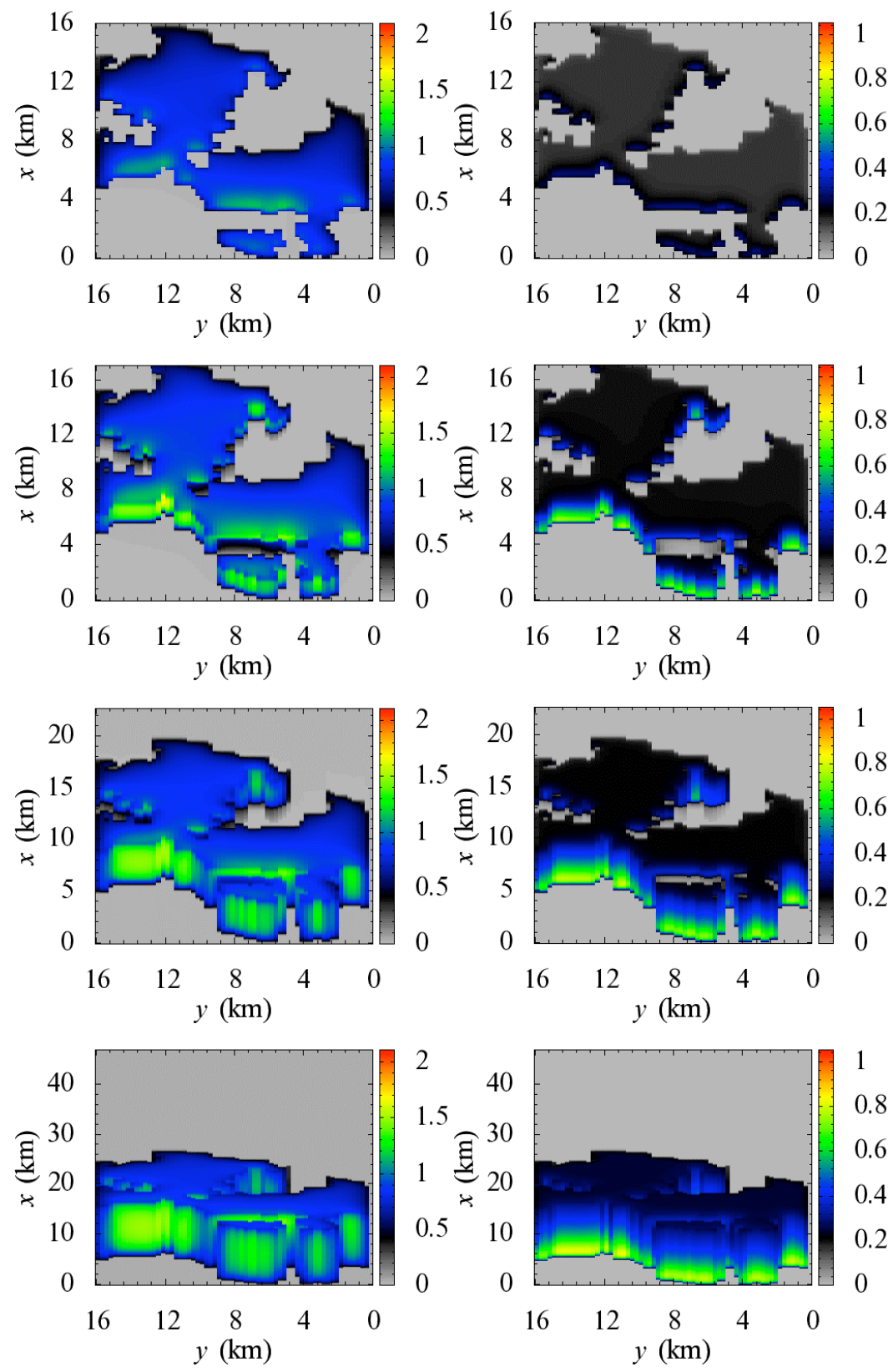

Fig. 5. Reflectance from a realization of a stochastic cloud model with constant cloud optical $(\tau=80)$ and geometrical thicknesses $(h=4 \mathrm{~km})$. Left column: $0.67 \mu \mathrm{m}$; right column: $2.1 \mu \mathrm{m} ; \theta_{0}=60^{\circ} ; \theta=0^{\circ}, 20^{\circ}, 45^{\circ}$, and $70^{\circ}$, from top to bottom. Note different color scales for left and right columns.

$x$ and $y$, with $z$ increasing from cloud base $z_{0}$ to cloud top $h(x, y)$, LWC and $r_{e}$ increase linearly, $N$ decreases as $z^{2}$, and $\sigma_{\text {ext }}$ is constant.

\section{Proof of concept}

Figure 5 shows an example of reflectances from a 16 by $16 \mathrm{~km}$ cloud field illuminated at $\theta_{0}=60^{\circ}$ [from South (bot- tom of the image)] and viewed at different viewing angles: $\theta$ $0^{\circ}, 20^{\circ}, 45^{\circ}$, and $70^{\circ}$ (also from South). The cloud is $4 \mathrm{~km}$ thick; for illustrative purposes, the cloud top is flat. Droplet effective radius grows linearly with height from 5 to $25 \mu \mathrm{m}$; thus in Eq. (3a), $a=5$ and $b=5 \mu \mathrm{m}$.

The two upper plots show nadir angle observations. As illustrated in Figs. 1 and 2, we see that at $0.67 \mu \mathrm{m}$ (left column), the cloud tops at the illuminated cloud edges are much brighter, whereas the cloud tops at the opposite ends look 
(a)

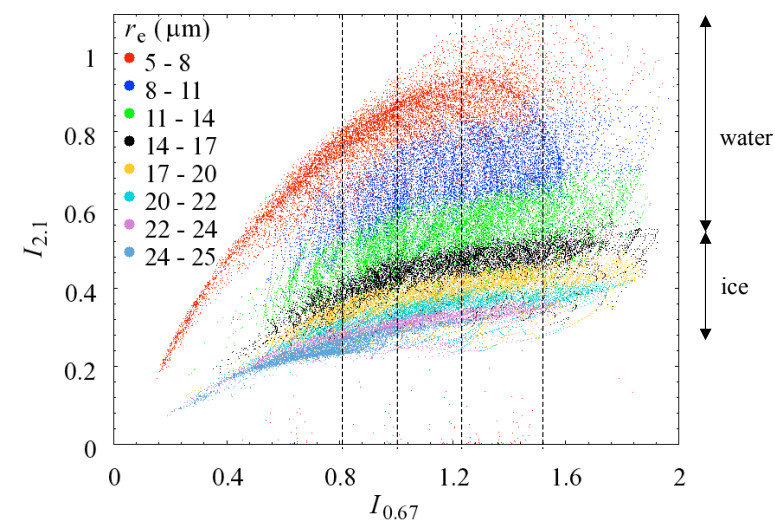

(b)
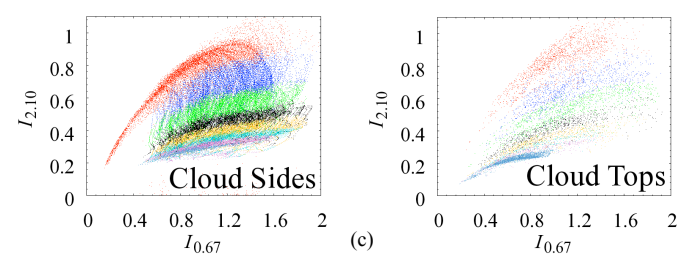

Fig. 6. A scatter plot of $2.1 \mu \mathrm{m}$ reflectances vs. $0.67 \mu \mathrm{m}$ reflectances based on 20 cloud fields generated by stochastic cloud model described in Sect. 3. Parameters of the model are the following: mean cloud optical thickness $=80$, mean cloud height $=$ $4 \mathrm{~km}$, spectral exponent $=2.0$, standard deviations $=16$ for the optical thickness and $1 \mathrm{~km}$ for the cloud height, cloud fraction $=0.5$, $\theta_{0}=60^{\circ}, \theta=70^{\circ}$. Particles smaller than $15 \mu \mathrm{m}$ are water droplets while particles larger than $15 \mu \mathrm{m}$ are ice. (a) Reflectances from both cloud sides and cloud tops. Dash lines indicate fixed $0.67 \mu \mathrm{m}$ reflectances $( \pm 0.03)$ used in Fig. 7. (b) Reflectance from cloud sides. (c) Reflectance from cloud tops.

darker then in the rest of the area. At $2.1 \mu \mathrm{m}$ (right column), cloud tops are homogeneous except may be the first $0.5 \mathrm{~km}$ away from the illuminated cloud edges. With increasing viewing angles, we start seeing illuminated cloud sides that are brighter than their cloud top counterparts. As a result, even visually one can distinguish between cloud sides and cloud tops, especially at low viewing angles. Similar to Fig. 2, at $0.67 \mu \mathrm{m}$ the reflectance from cloud sides reaches its maximum in the middle of the cloud while at $2.1 \mu \mathrm{m}$ the reflectance from cloud sides gradually decreases starting from about $0.5-1 \mathrm{~km}$ (10-20 optical depths) from the cloud base. This decrease is a clear signature of droplet sizes that are small $(5 \mu \mathrm{m})$ at the bottom and are large $(25 \mu \mathrm{m})$ and highly absorptive at the top.

A scatter plot on Fig. 6a is a Nakajima-King (1990) type diagram that relates cloud reflectances at 2.1 and $0.67 \mu \mathrm{m}$. The plot is based on 20 cloud fields generated as realizations of the stochastic cloud model described in Sect. 3. In contrast to a traditional Nakajima-King scatter plot that shows only the cloud-top reflectance, most of the points on Fig. 6a correspond to the reflectance from cloud sides. Indeed, pan- els (b) and (c) illustrate the break down of panel (a) into reflectance from cloud sides and cloud tops, respectively. Panel (b) is much brighter than panel (c), i.e., much more photons have been reflected from cloud sides than from cloud tops. We also see from panel (c) that, since cloud droplet (particle) size increases linearly with height (see, Eq. 3a), only those cloud tops that have the largest $r_{e}=25 \mu \mathrm{m}$ (blue dots) have substantially contributed to the total reflectance. Because of low VZA $\left(\theta=70^{\circ}\right)$, other cloud tops are in shadow and are barely seen by the observer. As explained in Sect. 2.3, at $2.1 \mu \mathrm{m}$ the cloud-top reflectances (blue dots) are the smallest. At $0.67 \mu \mathrm{m}$, the cloud-top reflectances have a wide range of values; the latter corresponds to the variety of cloud optical thicknesses as follows directly from the Nakajima-King (1990) theory.

Let us now fix the $0.67 \mu \mathrm{m}$ reflectances at four different levels (dash lines in Fig. 6a) and build histograms of $r_{e}$ for different values of the $2.1 \mu \mathrm{m}$ reflectances. Figure 7 illustrates them (with a window of \pm 0.03 for $I_{0.67}$ and \pm 0.02 for $I_{2.1}$ ). As expected, for $I_{0.67}=0.83$, most observed radiances are reflected from the cloud top with $r_{e}=25 \mu \mathrm{m}$. Increasing $I_{0.67}$, we observe more and more radiances reflected from the cloud side. For the large enough $0.67 \mu \mathrm{m}$ reflectances, $I_{2.1}$ saturates and, similar to the plane-parallel approximation, the retrieved values of $r_{e}$ become insensitive to the values of $I_{0.67}$. Because of the statistical nature of our retrievals, instead of a single value of $r_{e}$, we retrieve its (conditional) probability density, $p\left(r_{e} \mid I_{0.67}, I_{2.1}\right)$. The mean $r_{e}$ can be calculated as

$$
\left\langle r_{e}\right\rangle=\int_{0}^{\infty} r_{e} p\left(r_{e} \mid I_{0.67}, I_{2.1}\right) d r_{e}
$$

and its standard deviation $\sigma$ as

$\sigma=\sqrt{\int_{0}^{\infty}\left(r_{e}-\left\langle r_{e}\right\rangle\right)^{2} p\left(r_{e} \mid I_{0.67}, I_{2.1}\right) d r_{e}}$

For example, for $I_{0.67}=1.22 \pm 0.03$ and $I_{2.1}=0.58 \pm 0.02$, the mean retrieved value $\left\langle r_{e}\right\rangle=12 \mu \mathrm{m}$ with standard deviation $\sigma=2 \mu \mathrm{m}$.

If, in addition to the measurements at 0.67 and $2.1 \mu \mathrm{m}$, one also measures the cloud side brightness temperature, say at $11.6 \mu \mathrm{m}$, each retrieved distribution of effective radius can be directly related to cloud side brightness temperature, thus assessing its altitude. In other words, a combination of measurements at these three wavelengths can resolve the vertical distribution of cloud droplet sizes near cloud side. The extension of the retrieved profiles from cloud sides to the whole cloud requires an additional assumption of mild fluctuations of droplet effective radii along a horizontal plane at the same altitude $z$ inside clouds. As discussed in Martins et al. (2006) ${ }^{1}$, studies of in situ measurements in Cumulus clouds (e.g., Blyth and Latham, 1991) and cloud models (Zev Levin, private communications) confirm that this 

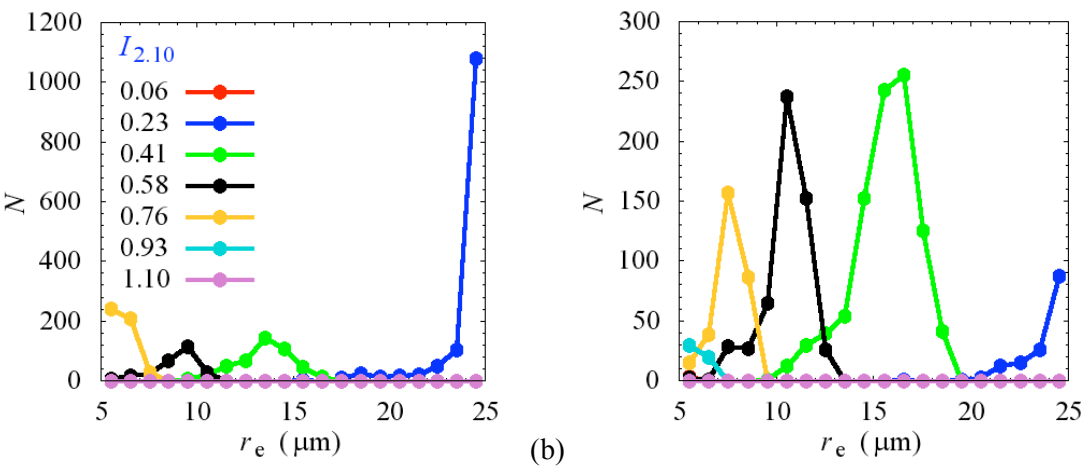

(a)

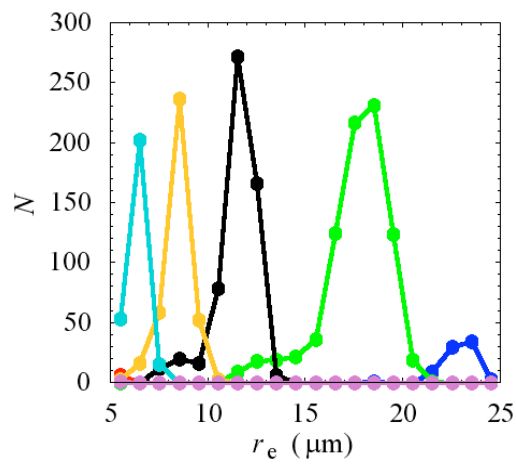

(b)

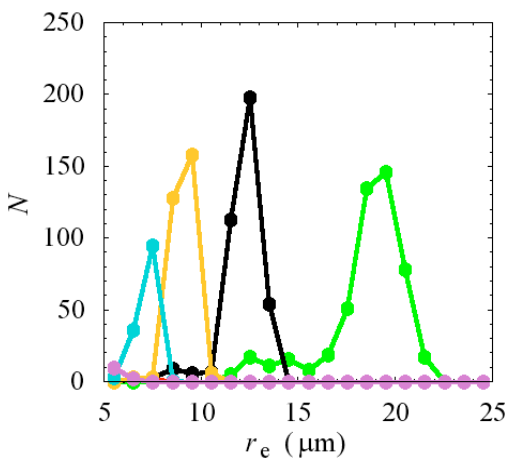

Fig. 7. Histograms (number of cases vs. effective radius) obtained from Fig. 6. Values of reflectance at $0.67 \mu \mathrm{m}$ were set to $0.83,1.03,1.22$, and 1.50 with a \pm 0.03 window, on panels (a), (b), (c) and (d) respectively. Values of reflectance at $2.1 \mu \mathrm{m}$ have a \pm 0.02 window.

assumption does not look unrealistic either. Rosenfeld and Lensky (1998) and Freud et al. (2005) extensively studied the temperature-effective radius vertical profiles for convective clouds. They found that cloud effective radius, to first approximation, can be assumed conservative for a given temperature.

Generally speaking, to retrieve a vertical profile of droplet effective radius, the above approach suggests using a database of stochastic cloud models and corresponding radiative transfer calculations of cloud reflectances at 0.67, 2.1 and $11.6 \mu \mathrm{m}$. This is similar to a Bayesian retrieval algorithm (e.g., McFarlane et al., 2002; Evans et al., 2002) that combines prior information about cloud structure and microphysics with radiative transfer calculations,

$p\left(\boldsymbol{x} \mid I_{0.67}, I_{2.1}, I_{11.6}\right)=\frac{p\left(I_{0.67}, I_{2.1}, I_{11.6} \mid \boldsymbol{x}\right) p(x)}{\int p\left(I_{0.67}, I_{2.1}, I_{11.6} \mid \boldsymbol{x}\right) p(x) d x}$. (8)

Here the vector $\boldsymbol{x}$ consist of cloud parameters (with $r_{e}$ ) that affect the cloud reflectances: $I_{0.67}, I_{2.1}$ and $I_{11.6}$. Function $p\left(I_{0.67}, I_{2.1}, I_{11.6} \mid x\right)$ is the conditional probability density function given vector $\boldsymbol{x}$. It is directly related to our pre-calculated database - the radiative transfer simulations of cloud reflectances for the cloud structure defined by $\boldsymbol{x}$. Figure 8 shows an example of the conditional probability function of two reflectances $I_{0.67}$ and $I_{2.1}$ for $r_{e}$ from 6 to $7 \mu \mathrm{m}$ and from 14 to $15 \mu \mathrm{m}$, respectively. Other parameters of cloud structure (vector $\boldsymbol{x}$ ) that affect calculations of $I_{0.67}$ and $I_{2.1}$ are described in Sect. 3 and in the caption to
Fig. 6. Note that the distribution functions in Fig. 8 are not necessarily Gaussian. Function $p(x)$ is the probability density function of cloud structure $\boldsymbol{x}$. In other words, based on the prior information it defines the weights to be accounted for characterizing the frequency of state $x$. The integral in the denominator on the right hand side of (7) is just a normalizing factor. Finally, the left hand side of (7) is the (posterior) probability density of having cloud structure $\boldsymbol{x}$ (including $r_{e}$ ) giving measurements of $I_{0.67}, I_{2.1}$ and $I_{11.6}$. It is related to histograms shown in Fig. 7. For details on a Bayesian retrieval algorithm applied to microwave radiometer and submillimeter-wave cloud ice radiometer see the excellent descriptions given in McFarlane et al. (2002) and Evans et al. (2002).

\section{Summary and discussion}

Knowledge on vertical distribution of droplet sizes is essential for understanding not only cloud development and precipitation but also the interactions between clouds and aerosols. Recently Andreae et al. (2004) using in situ aircraft measurements showed a strong dependence of various cloud properties (including droplet sizes), as a function of height in the cloud, on abundance of aerosol particles. How can one obtain this information globally from satellite remote sensing? 


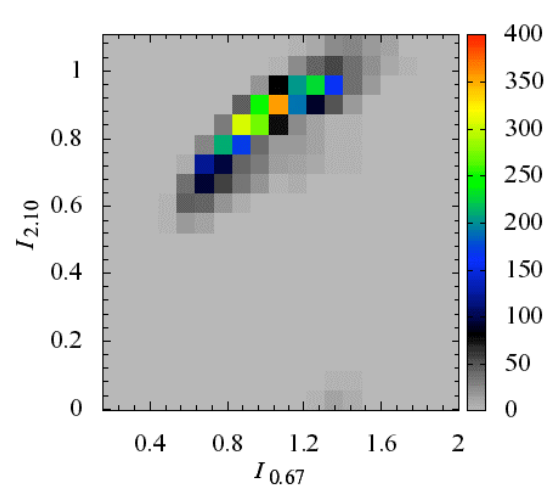

(a)

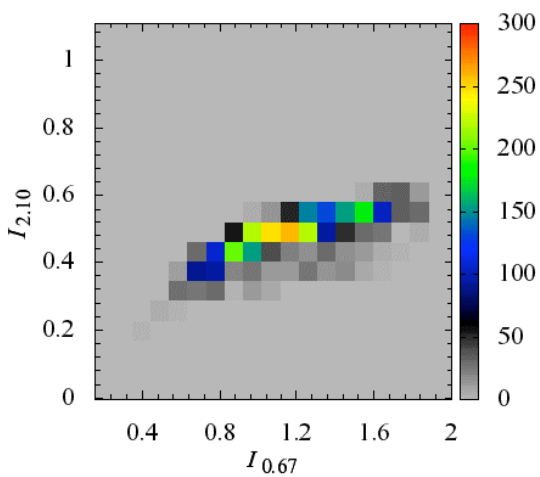

(b)

Fig. 8. Histograms of reflectances at $0.67 \mu \mathrm{m}$ and $2.1 \mu \mathrm{m}$ conditional the effective radius equal to (a) 6-7 $\mu \mathrm{m}$ and (b) $14-15 \mu \mathrm{m}$. Plot is based on 20 realizations of the stochastic cloud model described in Sect. 3. Parameters of the model are the same as in Fig. 6.

For this purpose, a new satellite mission, called CLAIM3D (stands for "3-D cloud aerosol interaction mission") has been recently proposed (Martins et al, 2006 ${ }^{1}$ ). The CLAIM$3 \mathrm{D}$ mission is designed to advance our understanding of cloud and precipitation development by measuring vertically resolved cloud parameters. It is proposed to have a unique combination of extended wavelength range $(0.38-12 \mu \mathrm{m})$, polarization, and multi-angle 3-D geometry combining the best features from POLDER (polarization and multi-angle), MISR (multi-angle), and MODIS (multi-channel) to characterize aerosols and cloud microphysics. This paper is the first step towards possible interpretation of CLAIM-3D measurements of reflected from cloud sides solar radiation.

Over the last two decades, considerable efforts have been dedicated to optical remote sensing of cloud properties. Using plane-parallel radiative transfer theory (e.g. Nakajima and King, 1990), measured radiances have been turned into science products, such as cloud optical depth and effective radius. If this approach is acceptable for a stratiform type clouds, it is suspect for clouds that are far from horizontally homogeneous (e.g., Varnai and Marshak, 2001; Iwabuchi and Hayasaka, 2002; Davis, 2002), especially for the clouds with a relatively small aspect ratio (the ratio of horizontal to vertical cloud dimensions) and well-developed cloud sides. These are the clouds the CLAIM-3D mission is directed for. In other words, here we target cloud side passive remote sensing rather than traditional cloud top remote sensing.

However, in order to interpret the cloud side measurements, a new 3-D-based cloud retrieval scheme should be developed. Advances in 3-D radiative transfer algorithms, improved understanding of 3-D cloud structure (Marshak and Davis, 2005), and increases in computing power make the time now ripe for 3-D cloud retrieval.

This paper studies the properties of radiation reflected from cloud sides at two wavelengths: one nonabsorbing $(0.67 \mu \mathrm{m})$ and one water-absorbing $(2.1 \mu \mathrm{m})$. As a proof of concept, it shows that (under some general assumptions and limitations) using Bayesian approach (e.g., Evans, 2002) simultaneous measurements of radiances at these two wavelengths can be mapped into a distribution of cloud droplet sizes. Not unlike the famous Nakajima-King (1990) diagram that maps cloud top reflections into a pair of cloud optical depth and effective radius, a new algorithm based on cloud stochastic models is capable in interpreting cloud side reflections at 0.67 and $2.1 \mu \mathrm{m}$ in terms of cloud droplet size distribution. If the information on cloud side brightness temperature is available, droplet size distributions can be vertically resolved.

Of course, knowledge of reflectance from the pixels surrounding each target pixel as well as reflectance at multiple angles will improve our retrieval making the width of the retrieved distribution narrower. However, to match cases in a simulated retrieval database with cloud side measurements we need to keep the number of parameters describing the relevant information about 3-D cloud structure as few as possible. As the next step, different combinations of radiances in our simulated retrieval database will be tested.

Obviously, the retrieved values of droplet effective radius will correspond to droplets located not far (less than $1 \mathrm{~km}$ ) from the cloud's outer walls. However, in situ observations (e.g., Freud et al., 2005) showed that the effective radius may remain constant for any given level in the cloud. In theses cases, retrieving effective radius near the cloud edges will give us information of the microphysics occurring in the cloud's core. These features are discussed in more details by Martins et al. (2006) ${ }^{1}$.

Acknowledgements. This work was supported by NASA Goddard Space Flight Center New Opportunities Office and by the U.S. Department of Energy as part of the Atmospheric Radiation Measurement (ARM) program. We thank H. Barker, A. Davis, G. Feingold, I. Koren, B. Mayer, L. Remer, T. Varnai, and G. Wen for stimulating discussions.

Edited by: Y. Rudich 


\section{References}

Andreae, M. O., Rosenfeld, D., Artaxo, P., Costa, A. A., Frank, G. P., Longo, K. M., and Silva-Dias, M. A. F.: Smoking rain clouds over the Amazon, Science, 303, 5662, 1337-1342, 2004.

Blyth, A. M. and Latham, J.: A climatological parameterization for cumulus clouds, J. Atmos. Sci., 48, 2367-2371, 2001.

Bréon, F. M. and Goloub, P.: Cloud droplet effective radius from spaceborne polarization measurements, Geophys. Res. Lett., 25, 1879-1882, 1998.

Bréon, F. M. and Doutriaux-Boucher, M.: A comparison of cloud droplet radii measured from space, IEEE Trans. Geosci. Remote Sens., 43(8), 1796-1805, 2005.

Chang, F.-L. and Li, Z.: Estimating the vertical variation of cloud droplet effective radius using multispectral near-infrared satellite measurements, J. Geophys. Res., 107(D15), 4257, doi:10.1029/2001JD000766, 2002.

Davies, R.: The effect of finite geometry on the three-dimensional transfer of solar irradiance in clouds, J. Atmos. Sci., 35, 17121725, 1978.

Davies, R.: Reflected solar radiances from broken cloud scenes and the interpretation of scanner measurements, J. Geophys. Res., 89, 1259-1266, 1984.

Davis, A. B.: Cloud remote sensing with sideways-looks: Theory and first results using Multispectral Thermal Imager (MTI) data, in: S.P.I.E. Proceedings, v. 4725, edited by: Shen, S. S. and Lewis, P. E., S.P.I.E. Publications, Bellingham (Wa), 2002.

Evans, K. F.: The Spherical Harmonics Discrete Ordinate Method for Three-Dimensional Atmospheric Radiative Transfer, J. Atmos. Sci., 55, 429-446, 1998.

Evans, K. F., Walter, S. J., Heymsfield, A. J., and McFarquhar, G. M.: The Submillimeter-wave cloud ice radiometer: Simulations of retrieval algorithm performance, J. Geophys. Res., 107, 4028, doi:10.1029/2001JD000709, 2002.

Evans K. F. and Marshak, A.: Numerical Methods in ThreeDimensional Radiative Transfer, in: Three-Dimensional Radiative Transfer in Cloudy Atmospheres, edited by: Marshak, A. and Davis, A. B., Springer, 243-282, 2005.

Freud, E., Rosenfeld, D., Andreae, M. O., Costa, A. A., and Artaxo, P.: Observed robust relations between $\mathrm{CCN}$ and vertical evolution of cloud drop size distributions in deep convective clouds, Atmos. Chem. Phys. Discuss., 5, 10 155-10 195, 2005.

Hirose, M. and Nakamura, K.: Spatiotemporal Variation of the Vertical Gradient of Rainfall Rate Observed by the TRMM Precipitation Radar, J. Clim., 17, 3378-3397, 2004.

Iwabuchi, H. and Hayasaka T.: Effects of cloud horizontal inhomogeneity on the optical thickness retrieved from moderateresolution satellite data, J. Atmos. Sci., 59, 2227-2242, 2002.

McFarlane, S. A., Evans, K. F., and Ackerman, A. S.: A Bayesian algorithm for the retrieval of liquid water properties from microwave radiometer and millimiter radar data, J. Geophys. Res., 107(D16), 4317, doi:10.1029/2001JD001011, 2002.

Marchuk, G., Mikhailov, G., Nazaraliev, M., Darbinjan, R., Kargin, B., and Elepov, B.: The Monte Carlo Methods in Atmospheric Optics, 208 pp., Springer-Verlag, New-York (NY), 1980.
Marshak, A., Davis, A., Cahalan, R. F., and Wiscombe, W. J.: Bounded cascade models as nonstationary multifractals, Phys. Rev. E, 49, 55-69, 1994.

Marshak, A., Davis, A., Wiscombe, W. J., Ridgway, W., and Cahalan, R. F.: Biases in shortwave column absorption in the presence of fractal clouds, J. Clim., 11, 431-446, 1998.

Marshak, A. and Davis, A. B. (Eds.): Three-Dimensional Radiative Transfer in Cloudy Atmospheres, Springer, 686 p, 2005.

McKee, T. B. and Cox, S. K.: Scattering of visible radiation by finite clouds, J. Atmos. Sci., 31, 1885-1892, 1974.

Nakajima, T. Y. and King, M. D.: Determination of the optical thickness and effective particle radius of clouds from reflected solar radiation measurements - Part I, Theory, J. Atmos. Sci., 47, 1878-1893, 1990.

Platnick, S.: Vertical photon transport in cloud remote sensing problems, J. Geophys. Res., 105, 22 919-22 935, 2000.

Platnick, S., King, M. D., Ackerman, S. A., Menzel, W. P., Baum, B. A., Riedi, J. C., and Frey, R. A.: The MODIS cloud products: Algorithms and examples from Terra, IEEE Trans. Geosci. Remote Sens., 41(2), 459-473, 2003.

Prigarin, S. and Marshak, A.: Numerical model of broken clouds adapted to observationsm, Atmos. Oceanic Opt., 18, 256-263, 2005.

Ramanathan, V., Crutzen, P. J., Kiehl, J. T., and Rosenfeld, D.: Aerosols, climate and the hydrological cycle, Science, 294, 2119-2124, 2001

Rosenfeld, D. and Lensky, I. M.: Satellite-based insights into precipitation formation processes in continental and maritime convective clouds, Bull. Am. Metereol. Soc., 79, 2457-2476, 1998.

Rosenfeld, D.: Suppression of rain and snow by urban and industrial air pollution, Science, 287, 1793-1796, 2000.

Rosenfeld, D. and Ulbrich, C. W.: Deep convective clouds with sustained supercooled liquid water down to $-37.5^{\circ} \mathrm{C}$, Nature, 405 , 440-442, 2000.

Schertzer, D. and Lovejoy, S.: Physical modeling and analysis of rain and clouds by anisotropic scaling multiplicative processes, J. Geophys. Res., 92, 9693-9714, 1987.

Stamnes, K., Tsay, S.-C., Wiscombe, W. J., and Jayaweera, K.: Numerically stable algorithm for discrete-ordinate-method radiative transfer in multiple scattering and emitting layered media, Appl. Opt., 27, 2502-2512, 1988.

Stephens, G. L.: Remote Sensing of the Lower Atmosphere. An Introduction, Oxford University Press, pp. 523, 1994.

Stephens, G. L., Vane, D., Boain, R., Mace, G., Sassen, K., Wang, Z., Illingworth, A., O'Connor, E., Rossow, W., Durden, S., Miller, S., Austin, R., Benedetti, A., Mitrescu, C., and CloudSat Science Team: The CloudSat mission and the A-Train: A new dimension of space-based observations of clouds and precipitation, Bull. Am. Metereol. Soc., 83, 1771-1790, 2002.

Várnai, T. and Marshak, A.: Observations and analysis of threedimensional radiative effects that influence MODIS cloud optical thickness retrievals, J. Atmos. Sci., 59, 1607-1618, 2002. 